\title{
Atomic data from the IRON Project. XV. Electron excitation of the fine-structure transitions in hydrogen-like ions He II and Fe XXVI
}

\author{
R. Kisielius ${ }^{\star}$, K.A. Berrington and P.H. Norrington \\ Department of Applied Mathematics \& Theoretical Physics, The Queen's University, Belfast, BT7 1NN, UK
}

Received October 17; accepted November 6, 1995

\begin{abstract}
Electron excitation collision strengths for fine-structure transitions involving relativistic levels with principal quantum number $n=1,2,3,4$ in hydrogen-like ions He II and Fe XxVI are calculated using R-matrix techniques. The model target ion includes all 25 energy levels with $n \leq 5$. Low-energy collision strengths dominated by autoionizing resonances are averaged over a Maxwellian velocity distribution to obtain effective collision strengths as a function of electron temperature. In the case of He II, such resonances enhance the collision strength by up to a factor of 3 over earlier calculations for excitation of the $n=4$ levels due to the presence of $5 \ln ^{\prime} l^{\prime}$ doubly-excited states near the $n=4$ thresholds. For He II, the data are presented at four temperature points ranging from $\log T(\mathrm{~K})=3.2$ to 4.3 , and for Fe XXVI from $\log T(\mathrm{~K})=6.0$ to 7.5 .
\end{abstract}

Key words: atomic data

\section{Introduction}

Emission lines of He II ions are observed in a wide range of astrophysical objects. The current mission of the Solar and Heliospheric Observatory (SOHO) will provide observations requiring reliable cross section data for interpretation. Iron is an important and abundant coronal element, and its lines have been observed from almost all ionization stages.

Considering He II ions, a number of theoretical data are available, especially for transitions $1 \mathrm{~s}-2 \mathrm{~s}$ and $1 \mathrm{~s}-2 \mathrm{p}$. The most consistent of them are results of Burke \& Taylor (1969), Morgan (1980), and Bray et al. (1993). Aggarwal et al. (1991) performed 15-state R-matrix nonrelativistic calculation of electron impact excitation within $n=1,2,3,4,5$ levels in He II ions. The method used is similar to the one presented in this paper but we use a relativistic approach giving additional information about excitation of fine-structure transitions between levels with the same principal quantum number $n$ as well as information about the excitation from relativistic levels. Although a statistical weighting approach or algebraic recoupling could be used to obtain information about excitation of relativistic levels from non-degenerate s-levels, it is not

Send offprint requests to: K.A. Berrington

* Permanent address: Institute of Theoretical Physics and Astronomy, A. Goštauto 12, 2600 Vilnius, Lithuania possible to get similar information about excitation from relativistic p-, d- levels.

One can find collision data for the excitation of transitions in Fe XXVI ions using parameters presented by Mann (1983), Golden et al. (1981), Clark et al. (1982). The two last papers contain very wide range of transitions including levels with principal quantum number $n \leq 5$. Unfortunately, these data are incomplete and inconsistent. First of all, they are presented for non-relativistic energy levels. Secondly, the parameters are obtained without accounting for autoionization resonance structure in collision strengths which give a significant contribution to effective collision strengths (Kisielius et al. 1995). Aggarwal \& Kingston (1993) overcome this inaccuracy by use of R-matrix techniques but restrict calculations for the nonrelativistic case only.

In present work we continue our earlier relativistic study of collisional excitation in hydrogen-like ions (Kisielius et al. 1995). The significant expansion of the previous calculation is to include energy levels with $n \leq 5$ in the target description which provides possibility to account for resonances in excitation collision strengths for transitions involving $n \leq 4$ levels.

The present calculation is part of the international collaboration known as the IRON Project (Hummer et al. 1993, referred to as Paper I) to obtain accurate collision 
rates for fine-structure transitions. Other papers in the IRON project series are listed in the references.

\section{The calculation}

The basic atomic theory, approximation and computer codes employed in the IRON Project are described in Paper I. In contrast to the Breit-Pauli approximation used in other papers of the IRON Project, we apply the fully relativistic Dirac R-matrix approach of Norrington \& Grant (1987) in the present calculation.

We include the 25 lowest energy levels $1 \mathrm{~s}_{1 / 2}, 2 \mathrm{~s}_{1 / 2}$, $2 \mathrm{p}_{1 / 2}, 2 \mathrm{p}_{3 / 2}, 3 \mathrm{~s}_{1 / 2}, 3 \mathrm{p}_{1 / 2}, 3 \mathrm{p}_{3 / 2}, 3 \mathrm{~d}_{3 / 2}, 3 \mathrm{~d}_{5 / 2}, 4 \mathrm{~s}_{1 / 2}, 4 \mathrm{p}_{1 / 2}$, $4 \mathrm{p}_{3 / 2}, 4 \mathrm{~d}_{3 / 2}, 4 \mathrm{~d}_{5 / 2}, 4 \mathrm{f}_{5 / 2}, 4 \mathrm{f}_{7 / 2}, 5 \mathrm{~s}_{1 / 2}, 5 \mathrm{p}_{1 / 2}, 5 \mathrm{p}_{3 / 2}, 5 \mathrm{~d}_{3 / 2}$, $5 \mathrm{~d}_{5 / 2}, 5 \mathrm{f}_{5 / 2}, 5 \mathrm{f}_{7 / 2}, 5 \mathrm{~g}_{7 / 2}, 5 \mathrm{~g}_{9 / 2}$ to describe the ionic target in collision process. The target wavefunctions are generated using the GRASP program of Grant et al. (1980) in which a single-particle Dirac Hamiltonian accounting for mass-correction, Darwin and spin-orbit terms with the Coulomb electron-electron interaction is employed. In the non-relativistic approximation, the levels of hydrogen atoms and hydrogen-like ions are quasidegenerate, i.e. the levels with the same principal quantum number $n$ and different orbital angular momentum $l$ have the same energy. In the relativistic case, this is true for levels with the same $n$ and total angular momentum $J$. These levels split due to inclusion of radiative corrections (Lamb shift).

Table 1. Energy levels (in Ry) of He II and Fe XXVI ions

\begin{tabular}{r|rrr}
\hline Index & Level & He II & Fe XXVI \\
\hline 1 & $1 \mathrm{~s}_{1 / 2}$ & 0.000000000 & 0.0000000 \\
2 & $2 \mathrm{p}_{1 / 2}$ & 3.000113769 & 510.9519822 \\
3 & $2 \mathrm{~s}_{1 / 2}$ & 3.000118044 & 510.9949940 \\
4 & $2 \mathrm{p}_{3 / 2}$ & 3.000167149 & 512.5109778 \\
5 & $3 \mathrm{p}_{1 / 2}$ & 3.555712294 & 606.0908759 \\
6 & $3 \mathrm{~s}_{1 / 2}$ & 3.555713560 & 606.1038432 \\
7 & $3 \mathrm{~d}_{3 / 2}$ & 3.555728079 & 606.5523105 \\
8 & $3 \mathrm{p}_{3 / 2}$ & 3.555728106 & 606.5531434 \\
9 & $3 \mathrm{~d}_{5 / 2}$ & 3.555733356 & 606.7038123 \\
10 & $4 \mathrm{p}_{1 / 2}$ & 3.750169601 & 639.3257232 \\
11 & $4 \mathrm{~s}_{1 / 2}$ & 3.750170139 & 639.3311999 \\
12 & $4 \mathrm{~d}_{3 / 2}$ & 3.750176263 & 639.5202350 \\
13 & $4 \mathrm{p}_{3 / 2}$ & 3.750176272 & 639.5205885 \\
14 & $4 \mathrm{f}_{5 / 2}$ & 3.750178487 & 639.5840838 \\
15 & $4 \mathrm{~d}_{5 / 2}$ & 3.750178487 & 639.5841977 \\
16 & $4 \mathrm{f}_{7 / 2}$ & 3.750179599 & 639.6159335 \\
\hline
\end{tabular}

Relativistic separation of energy levels, particularly in the He II ions, is very small (see Table 1). In order to avoid inaccuracies arising from restriction of the target wavefunction expansion, we adjust the target energies by incorporating high-precision theoretical data of Erickson (1977) in the internal region matrix before diagonalization. These energy levels, along with the level indexes for He II ions, are presented in Table 1.
Total collision strengths for transitions in hydrogenlike ions are determined as a sum of partial collision strengths with particular angular momentum $J_{\text {tot }}$ of the system atom+electron. We calculate partial collision strengths for $J_{\text {tot }} \leq 40$ to achieve convergence of optically allowed transitions between the $n=3$ and $n=4$ levels as well as for transitions between energy levels with the same $n$ values. For transitions between quasidegenerate levels we add 'top-up' values to account for partial collision strengths with $J_{\text {tot }}>40$ by assuming a geometrical series and summing to infinity.

We perform the calculation for Fe XXVI using the same framework. In Table 1, we present accurate theoretical energy level values from Erickson (1977) used in our calculation. One can notice considerably larger energy splitting compared with that for He II ions. This is due to the increased relativistic corrections. Nevertheless, energy levels with the same $n$ and $J$ values lie rather close, so we have to account for high $J_{\text {tot }}$ partial collision strengths by 'topup', as above.

\section{Results}

Collision strengths are computed for each ion for the transitions including levels up to $n=4$ over a wide and sufficiently fine energy mesh. This enables us to integrate the data over a Maxwellian distribution of electron velocities to determine the effective collision strength $\Upsilon$. Having tabulated $\Upsilon$ values, one can easily obtain electron excitation and de-excitation rate coefficients.

Table 2 provides effective collision strengths for He II at single temperature point $T=10000 \mathrm{~K}$ obtained using various approximations. In our case, the collision strengths $\Omega$ are calculated in the energy region from 3.004 Ry to 7.0 Ry. In the interval $E=3.34-3.82 \mathrm{Ry}$ where resonance structure is very rich, $\Omega$ are calculated at more than 5500 energy points providing fairly correct information in the resonance regions. We sum our effective collision strengths over all possible $J_{\text {tot }}$ values in order to compare with available non-relativistic data.

With some exceptions, there is reasonably good agreement of our data with the previous calculation of Aggarwal et al. (1991) where a non-relativistic R-matrix technique was used. However, differences of up to a factor of 3 exist for the excitation of $n=4$ levels. This is because in the earlier calculation of $\Omega$, the energy mesh was not fine enough to get full information about the resonance structure. This could lead to substantial error in determining effective collision strengths because the lowest resonances, corresponding to $5 \ln ^{\prime} l^{\prime}$ doubly excited states of helium, lie very close to $n=4$ excitation thresholds (Herrick \& Sinanoğlu 1975). These resonances become very significant at low temperatures where the value of the effective collision strength $\Upsilon$ depends on the near-threshold collision strengths. The agreement with data of Hummer \& Storey (1987) is worse (with exception of $1 s-2 s$ and $1 s-2 p$ 
Table 2. Comparison of calculated effective collision strengths $\Upsilon$ with non-relativistic data for some transitions in $\mathrm{He}$ II at temperature $10^{4} \mathrm{~K}$. DRM denotes present relativistic 25-state Dirac R-matrix calculation, NRM denotes 15-state non-relativistic R-matrix calculation data of Aggarwal et al. (1991), and HS denotes data obtained using formulae from Hummer \& Storey (1987)

\begin{tabular}{c|ccc}
\hline Transition & DRM & NRM & HS \\
\hline $1 \mathrm{~s}-2 \mathrm{~s}$ & $1.67-1$ & $1.56-1$ & $1.42-1$ \\
$1 \mathrm{~s}-2 \mathrm{p}$ & $3.58-1$ & $3.46-1$ & $3.16-1$ \\
$1 \mathrm{~s}-3 \mathrm{~s}$ & $4.67-2$ & $3.88-2$ & $5.61-2$ \\
$1 \mathrm{~s}-3 \mathrm{p}$ & $7.26-2$ & $6.08-2$ & $9.53-2$ \\
$1 \mathrm{~s}-3 \mathrm{~d}$ & $4.70-2$ & $3.96-2$ & $6.06-2$ \\
$1 \mathrm{~s}-4 \mathrm{~s}$ & $2.56-2$ & $8.63-3$ & \\
$1 \mathrm{~s}-4 \mathrm{p}$ & $4.25-2$ & $1.55-2$ & \\
$1 \mathrm{~s}-4 \mathrm{~d}$ & $3.06-2$ & $1.56-2$ & \\
$1 \mathrm{~s}-4 \mathrm{f}$ & $9.50-3$ & $8.43-3$ & \\
$2 \mathrm{~s}-3 \mathrm{~s}$ & $5.89-1$ & $5.95-1$ & $6.34-1$ \\
$2 \mathrm{~s}-3 \mathrm{p}$ & $2.06+0$ & $1.61+0$ & $2.30+0$ \\
$2 \mathrm{~s}-3 \mathrm{~d}$ & $1.80+0$ & $1.56+0$ & $2.33+0$ \\
$2 \mathrm{p}-3 \mathrm{~s}$ & $2.12+0$ & $1.67+0$ & $2.26+0$ \\
$2 \mathrm{p}-3 \mathrm{p}$ & $5.18+0$ & $4.62+0$ & $6.15+0$ \\
$2 \mathrm{p}-3 \mathrm{~d}$ & $1.23+1$ & $1.04+1$ & $1.38+1$ \\
$3 \mathrm{~s}-3 \mathrm{~d}$ & $2.68+1$ & $2.94+1$ & \\
$3 \mathrm{~s}-4 \mathrm{~s}$ & $2.14+0$ & $1.37+0$ & \\
$3 \mathrm{~s}-4 \mathrm{p}$ & $5.75+0$ & $2.90+0$ & \\
$4 \mathrm{~s}-4 \mathrm{~d}$ & $5.53+1$ & $7.45+1$ & \\
$4 \mathrm{~s}-4 \mathrm{f}$ & $3.88+1$ & $2.48+1$ & \\
\hline
\end{tabular}

transitions). This can be explained by fact that data used to get fitting parameters in their work were taken from old calculations which did not account for a higher excited states such as $n=4,5$.

The effective collision strengths for some transitions in Fe XxvI ions obtained using various approximations are presented in Table 3 . In our R-matrix calculation, the energy interval is chosen to be from 512.5 Ry to $1000 \mathrm{Ry}$. This enables us to obtain $\Upsilon$ for temperature $T \leq 310^{7} \mathrm{~K}$. We use an energy mesh containing more than 1500 points with a high density in the resonance areas. This enables us to avoid uncertainties in performing the integration to obtain effective collision strengths $\Upsilon$.

One can see from Table 3 that agreement with the data of Aggarwal and Kingston (1993) is very good in general, though less good for $1 \mathrm{~s}-4 l$. The comparison with the data obtained using Coulomb-Born-Oppenheimer (CBO) approximation (Golden et al. 1981; Clark et al. 1982) makes it clear that the effect of autoionizing resonances becomes more and more significant for excitation to a higher levels. One can notice very good agreement in the case of $1 \mathrm{~s}-2 \mathrm{~s}$ and $1 \mathrm{~s}-2 \mathrm{p}$ excitation, but much poorer in the case of excitation to the $n=3$ and $n=4$ states. A similar statement is valid for the data of Mann (1983). It should be noted that agreement between two last columns in Table 3 in-
Table 3. Comparison of calculated effective collision strengths $\Upsilon$ with non-relativistic calculation data for some transitions in Fe XXVI at temperature $10^{6} \mathrm{~K}$. DRM denotes present relativistic 25-state Dirac R-matrix calculation, NRM denotes 15-state non-relativistic R-matrix calculation data (Aggarwal \& Kingston 1993), CBO denotes data obtained using Coulomb-Born-Oppenheimer approximation (Golden et al. 1981; Clark et al. 1982), and DWX denotes distorted wave calculation (Mann 1983)

\begin{tabular}{c|cccc}
\hline Transition & DRM & NRM & CBO & DWX \\
\hline $1 \mathrm{~s}-2 \mathrm{~s}$ & $1.14-3$ & $1.02-3$ & $1.07-3$ & $1.10-3$ \\
$1 \mathrm{~s}-2 \mathrm{p}$ & $4.52-3$ & $4.29-3$ & $4.39-3$ & $4.34-3$ \\
$1 \mathrm{~s}-3 \mathrm{~s}$ & $2.79-4$ & $2.69-4$ & $2.27-4$ & $2.32-4$ \\
$1 \mathrm{~s}-3 \mathrm{p}$ & $1.04-3$ & $1.03-3$ & $9.34-4$ & $9.36-4$ \\
$1 \mathrm{~s}-3 \mathrm{~d}$ & $2.79-4$ & $2.69-4$ & $1.57-4$ & $1.57-4$ \\
$1 \mathrm{~s}-4 \mathrm{~s}$ & $1.63-4$ & $9.73-5$ & $8.63-5$ & $8.77-5$ \\
$1 \mathrm{~s}-4 \mathrm{p}$ & $4.67-4$ & $3.93-4$ & $3.56-4$ & $3.60-4$ \\
$1 \mathrm{~s}-4 \mathrm{~d}$ & $1.50-4$ & $1.36-4$ & $8.04-5$ & $8.01-5$ \\
$1 \mathrm{~s}-4 \mathrm{f}$ & $4.77-5$ & $6.59-5$ & $5.21-6$ & $5.20-6$ \\
$2 \mathrm{~s}-3 \mathrm{~s}$ & $1.26-2$ & $1.23-2$ & $1.31-2$ & \\
$2 \mathrm{~s}-3 \mathrm{p}$ & $1.45-2$ & $1.56-2$ & $1.34-2$ & \\
$2 \mathrm{~s}-3 \mathrm{~d}$ & $2.76-2$ & $3.15-2$ & $2.66-2$ & \\
$2 \mathrm{p}-3 \mathrm{~s}$ & $5.88-3$ & $5.41-3$ & $2.68-3$ & \\
$2 \mathrm{p}-3 \mathrm{p}$ & $5.87-2$ & $6.30-2$ & $5.07-2$ & \\
$2 \mathrm{p}-3 \mathrm{~d}$ & $1.73-1$ & $1.87-1$ & $1.64-1$ & \\
$3 \mathrm{~s}-3 \mathrm{~d}$ & $2.33-1$ & $2.12-1$ & & \\
$3 \mathrm{~s}-4 \mathrm{~s}$ & $5.83-2$ & $5.62-2$ & $5.92-2$ & \\
$3 \mathrm{~s}-4 \mathrm{p}$ & $3.89-2$ & $4.03-2$ & $3.26-2$ & \\
$4 \mathrm{~s}-4 \mathrm{~d}$ & $8.42-1$ & $8.12-1$ & & \\
4s - 4f & $2.04-1$ & $1.92-1$ & &
\end{tabular}

dicates validity of $\mathrm{CBO}$ approximation for hydrogen-like ions with high $Z$.

We present effective collision strengths $\Upsilon$ at four electron temperatures in Tables 4 and 5, for transitions between relativistic energy levels with principal quantum number $n \leq 4$. Effective collision strengths for He II are presented in Table 4, and for Fe Xxvi are in Table 5 . In addition, the quasi-degenerate $2 \mathrm{~s}_{1 / 2}-2 \mathrm{p}_{3 / 2}$ transition was discussed in Kisielius et al. (1995). Due to the large amount of transitions it is not feasible to present $\Upsilon$ at more temperature points in this paper. However, one can obtain values of effective collision strengths from the IRON Project database for temperatures other than those presented in Tables 4 and 5. Alternatively, one can get approximate $\Upsilon$ values from these tables using interpolation procedures. The maximum temperature quoted here should be regarded as the limit to the validity of the present calculation.

\section{Discussion}

Collision strengths have been calculated using relativistic R-matrix techniques for excitation of fine structure transitions involving $n=1,2,3,4$ levels in hydrogen-like ions 
He II and Fe XXvi. This is the first such calculation on H-like systems, so it is not possible to compare with earlier relativistic calculations. Comparison with non-relativistic calculations demonstrates a good agreement for most lowlying transitions, but point to the importance of delineating resonance structure, particularly at low temperatures in He II.

A particular difficulty with calculations on atoms and near-neutral ions is the effect of ionization channels at intermediate energies. We omit such channels, as did Aggarwal et al. (1991), but note that work on the intermediate energy R-matrix method (IERM) by Odgers et al. (1995) indicates that this omission could lead to an overestimation of around $10 \%$ in our effective collision strengths for He II at the higher temperatures.

Acknowledgements. This work was done with the support of PPARC grant GR/H93576 and an EC network contract ERB CHRX CT920013. The calculations were carried out using CRAY Y-MP/EL computer at the Queen's University of Belfast Computer Centre. One of us (RK) acknowledges gratefully The Royal Society postdoctoral fellowship.

\section{References}

Aggarwal K.M., Berrington K.A, Kingston K.A., Pathak A., 1991, J. Phys. B 24, 1757

Aggarwal K.M., Kingston K.A., 1993, ApJS 85, 187

Bautista M.A., Pradhan A.K., 1995, A\&AS (in press) (Paper XIII)

Berrington K.A., 1995, A\&AS 109, 193 (Paper VIII)

Bray I., McCarthy I.E., Wigley J., Stelbovics A.T., 1993, J. Phys. B 26, L831

Burke P.G., Taylor A.J., 1969, J. Phys. B 2, 44

Butler K., Zeippen C.J., 1994, A\&AS 108, 1 (Paper V)

Clark R.E.H., Sampson D.H., Goett S.J., 1982, ApJS 49, 545

Erickson G.W., 1977, J. Chem. Phys. Ref. Data 6, 601

Galavis M.E., Mendoza C., Zeippen C.J., 1995, A\&AS 111, 347 (Paper X)

Golden L.B., Clark R.E.H., Goett S.J., Sampson D.H., 1981, ApJS 45, 603

Grant I., McKenzie B.J., Norrington P.H., Mayers D.F., Pyper N.C., 1980, Comp. Phys. Commun. 21, 207

Herrick D.R., Sinanoğlu O., 1975, Phys. Rev. A11, 97

Hummer D.G., Berrington K.A., Eissner W., Pradhan A.K., Saraph H.E., Tully J.A., A\&A 279, 298 (Paper I)

Hummer D.G., Storey P.J., 1987, MNRAS 224, 801

Kisielius R., Berrington K.A., Norrington P.H., 1995, J. Phys. B 28, 2459

Lennon D.J., Burke V.M., 1994, A\&AS 103, 273 (Paper II)

Mann J.B., 1983, Atom. Data Nucl. Data Tables 29, 407

Nahar S.N., 1994, A\&A 293, 967 (Paper VII)

Morgan L.A., 1980, J. Phys. B 13, 3703

Norrington P.H., Grant I.P., 1987, J. Phys. B 20, 4869

Pelan J.C., Berrington K.A., 1995, A\&AS 110, 209 (Paper IX)

Pelan J.C., Berrington K.A., 1995, A\&AS (in press) (Paper XII)

Odgers B.R., Scott M.P., Burke P.G., 1995, J. Phys. B 28, 2973

Saraph H.E., Tully J.A., 1994, A\&AS, 107, 29 (Paper IV)
Saraph H.E., Storey P.J., 1995, A\&AS (in press) (Paper XI) Storey P.J., Mason H.E., Saraph H.E., 1995, A\&AS (in press) (Paper XIV)

Zhang H.L., Graziani M., Pradhan A.K., 1994, A\&A 283, 319 (Paper III)

Zhang H.L., Pradhan A.K., 1994, A\&A 293, 953 (Paper VI)

Table 4. Effective collision strengths for finestructure transitions in He II ions at four temperature points $\log T(\mathrm{~K})=3.2,3.5,4.0,4.3$. Line notation corresponds to the level indexes presented in Table 1

\begin{tabular}{|c|c|c|c|c|c|}
\hline & line & 3.2 & 3.5 & 4.0 & 4.3 \\
\hline 1 & 2 & $1.12(-1)$ & $1.14(-1)$ & $1.20(-1)$ & $1.26(-1)$ \\
\hline 1 & 3 & $1.76(-1)$ & $1.73(-1)$ & $1.67(-1)$ & $1.62(-1)$ \\
\hline 1 & - & $2.21(-1)$ & $2.26(-1)$ & $2.38(-1)$ & $2.52(-1)$ \\
\hline 1 & - & $2.35(-2)$ & $2.27(-2)$ & $2.43(-2)$ & $2.57(-2)$ \\
\hline 1 & _ & $5.04(-2)$ & $4.74(-2)$ & $4.67(-2)$ & $4.55(-2)$ \\
\hline 1 & - & $2.22(-2)$ & $2.05(-2)$ & $1.88(-2)$ & $1.76(-2)$ \\
\hline 1 & - & $4.60(-2)$ & $4.47(-2)$ & $4.83(-2)$ & $5.12(-2)$ \\
\hline 1 & - & $3.35(-2)$ & $3.09(-2)$ & $2.82(-2)$ & $2.64(-2)$ \\
\hline 1 & $-\quad 10$ & $2.10(-2)$ & $1.76(-2)$ & $1.42(-2)$ & $1.39(-2)$ \\
\hline 1 & - 11 & $3.63(-2)$ & $3.11(-2)$ & $2.56(-2)$ & $2.44(-2)$ \\
\hline 1 & - 12 & $1.81(-2)$ & $1.53(-2)$ & $1.22(-2)$ & $1.12(-2)$ \\
\hline 1 & - 13 & $4.15(-2)$ & $3.48(-2)$ & $2.83(-2)$ & $2.77(-2)$ \\
\hline 1 & - 14 & $5.11(-3)$ & $4.81(-3)$ & $4.08(-3)$ & $3.34(-3)$ \\
\hline 1 & $-\quad 15$ & $2.73(-2)$ & $2.31(-2)$ & $1.84(-2)$ & $1.68(-2)$ \\
\hline 1 & - 16 & $6.82(-3)$ & $6.40(-3)$ & $5.42(-3)$ & $4.43(-3)$ \\
\hline 2 & - & $8.04(+0)$ & $7.73(+0)$ & $6.93(+0)$ & $6.40(+0)$ \\
\hline 2 & - & $9.40(-1)$ & $9.02(-1)$ & $8.85(-1)$ & $8.95(-1)$ \\
\hline 2 & - & $8.74(-1)$ & $8.05(-1)$ & $6.96(-1)$ & $6.20(-1)$ \\
\hline 2 & - & $2.52(+0)$ & $2.57(+0)$ & $2.84(+0)$ & $3.18(+0)$ \\
\hline 2 & - & $1.03(+0)$ & $9.42(-1)$ & $8.40(-1)$ & $7.65(-1)$ \\
\hline 2 & - & $1.06(+0)$ & $1.07(+0)$ & $1.16(+0)$ & $1.18(+0)$ \\
\hline 2 & -10 & $4.54(-1)$ & $4.06(-1)$ & $3.37(-1)$ & $3.16(-1)$ \\
\hline 2 & - 11 & $4.30(-1)$ & $3.96(-1)$ & $3.19(-1)$ & $2.70(-1)$ \\
\hline 2 & - 12 & $6.58(-1)$ & $6.32(-1)$ & $6.44(-1)$ & $7.34(-1)$ \\
\hline 2 & - 13 & $6.31(-1)$ & $5.51(-1)$ & $4.35(-1)$ & $3.70(-1)$ \\
\hline 2 & - 14 & $5.46(-1)$ & $5.68(-1)$ & $6.00(-1)$ & $5.93(-1)$ \\
\hline 2 & $-\quad 15$ & $5.75(-1)$ & $5.19(-1)$ & $4.53(-1)$ & $4.12(-1)$ \\
\hline 2 & - 16 & $3.03(-1)$ & $3.12(-1)$ & $3.43(-1)$ & $3.13(-1)$ \\
\hline 3 & 5 & $7.65(-1)$ & $7.22(-1)$ & $6.89(-1)$ & $6.84(-1)$ \\
\hline 3 & 6 & $5.74(-1)$ & $5.52(-1)$ & $5.89(-1)$ & $6.50(-1)$ \\
\hline 3 & - & $7.12(-1)$ & $6.97(-1)$ & $7.15(-1)$ & $7.54(-1)$ \\
\hline 3 & - & $1.51(+0)$ & $1.43(+0)$ & $1.37(+0)$ & $1.36(+0)$ \\
\hline 3 & 9 & $1.08(+0)$ & $1.05(+0)$ & $1.08(+0)$ & $1.13(+0)$ \\
\hline 3 & -10 & $3.48(-1)$ & $3.05(-1)$ & $2.52(-1)$ & $2.39(-1)$ \\
\hline 3 & - 11 & $3.96(-1)$ & $3.40(-1)$ & $2.64(-1)$ & $2.37(-1)$ \\
\hline 3 & - 12 & $3.96(-1)$ & $3.57(-1)$ & $2.91(-1)$ & $2.67(-1)$ \\
\hline 3 & - 13 & $6.90(-1)$ & $6.06(-1)$ & $5.02(-1)$ & $4.77(-1)$ \\
\hline 3 & - 14 & $2.05(-1)$ & $2.04(-1)$ & $2.00(-1)$ & $1.95(-1)$ \\
\hline 3 & $-\quad 15$ & $5.96(-1)$ & $5.36(-1)$ & $4.37(-1)$ & $4.01(-1)$ \\
\hline 3 & - 16 & $2.72(-1)$ & $2.72(-1)$ & $2.66(-1)$ & $2.59(-1)$ \\
\hline
\end{tabular}


Table 4. continued

\begin{tabular}{|c|c|c|c|c|c|}
\hline & line & 3.2 & 3.5 & 4.0 & 4.3 \\
\hline$\overline{4}$ & 5 & $1.03(+0)$ & $9.47(-1)$ & $8.39(-1)$ & $7.64(-1)$ \\
\hline 4 & 6 & $1.76(+0)$ & $1.63(+0)$ & $1.42(+0)$ & $1.28(+0)$ \\
\hline 4 & 7 & $1.90(+0)$ & $1.91(+0)$ & $2.05(+0)$ & $2.15(+0)$ \\
\hline 4 & 8 & $2.90(+0)$ & $2.75(+0)$ & $2.62(+0)$ & $2.57(+0)$ \\
\hline 4 & 9 & $5.42(+0)$ & $5.61(+0)$ & $6.27(+0)$ & $6.95(+0)$ \\
\hline 4 & -10 & $6.40(-1)$ & $5.59(-1)$ & $4.34(-1)$ & $3.65(-1)$ \\
\hline 4 & 11 & $8.78(-1)$ & $8.08(-1)$ & $6.48(-1)$ & $5.48(-1)$ \\
\hline 4 & 12 & $8.50(-1)$ & $7.77(-1)$ & $7.09(-1)$ & $6.81(-1)$ \\
\hline 4 & 13 & $1.57(+0)$ & $1.40(+0)$ & $1.16(+0)$ & $1.05(+0)$ \\
\hline 4 & 14 & $7.28(-1)$ & $7.47(-1)$ & $8.55(-1)$ & $8.25(-1)$ \\
\hline 4 & 15 & $1.69(+0)$ & $1.59(+0)$ & $1.56(+0)$ & $1.67(+0)$ \\
\hline 4 & 16 & $1.15(+0)$ & $1.20(+0)$ & $1.27(+0)$ & $1.23(+0)$ \\
\hline 5 & 8 & $1.47(+1)$ & $1.46(+1)$ & $1.53(+1)$ & $1.55(+1)$ \\
\hline 5 & 9 & $8.90(+0)$ & $8.57(+0)$ & $7.80(+0)$ & $6.89(+0)$ \\
\hline 5 & 10 & $2.29(+0)$ & $2.12(+0)$ & $2.06(+0)$ & $2.45(+0)$ \\
\hline 5 & 11 & $1.85(+0)$ & $1.72(+0)$ & $1.49(+0)$ & $1.36(+0)$ \\
\hline 5 & 12 & $7.26(+0)$ & $6.85(+0)$ & $6.46(+0)$ & $6.80(+0)$ \\
\hline 5 & 13 & $3.04(+0)$ & $2.68(+0)$ & $2.21(+0)$ & $1.96(+0)$ \\
\hline 5 & 14 & $3.86(+0)$ & $3.85(+0)$ & $4.11(+0)$ & $4.49(+0)$ \\
\hline 5 & 15 & $3.13(+0)$ & $2.88(+0)$ & $2.60(+0)$ & $2.79(+0)$ \\
\hline 5 & 16 & $2.16(+0)$ & $2.18(+0)$ & $2.17(+0)$ & $2.01(+0)$ \\
\hline 6 & 7 & $5(+1)$ & $1.05(+1)$ & $7(+1)$ & $.06(+1)$ \\
\hline 6 & 9 & $1.57(+1)$ & $1.58(+1)$ & $1.61(+1)$ & $1.60(+1)$ \\
\hline 6 & - 10 & $2.40(+0)$ & $2.19(+0)$ & $1.91(+0)$ & $1.81(+0)$ \\
\hline 6 & 11 & $2.59(+0)$ & $2.28(+0)$ & $2.14(+0)$ & $2.49(+0)$ \\
\hline 6 & 12 & $2.73(+0)$ & $2.56(+0)$ & $2.37(+0)$ & $2.48(+0)$ \\
\hline 6 & 13 & $4.79(+0)$ & $4.38(+0)$ & $3.84(+0)$ & $3.65(+0)$ \\
\hline 6 & 14 & $2.42(+0)$ & $2.47(+0)$ & $2.72(+0)$ & $3.19(+0)$ \\
\hline 6 & 15 & $4.12(+0)$ & $3.84(+0)$ & $3.41(+0)$ & $3.25(+0)$ \\
\hline 6 & 16 & $3.19(+0)$ & $3.20(+0)$ & $3.31(+0)$ & $3.51(+0)$ \\
\hline 7 & - & 1) & 1) & & $1)$ \\
\hline 7 & 10 & $4.58(+0)$ & $4.28(+0)$ & $3.65(+0)$ & $3.23(+0)$ \\
\hline 7 & 11 & $3.10(+0)$ & $2.86(+0)$ & $2.23(+0)$ & $1.76(+0)$ \\
\hline 7 & 12 & $7.90(+0)$ & $7.62(+0)$ & $7.39(+0)$ & $7.94(+0)$ \\
\hline 7 & 13 & $4.79(+0)$ & $4.44(+0)$ & $3.62(+0)$ & $2.96(+0)$ \\
\hline 7 & 14 & $1.74(+1)$ & $1.83(+1)$ & $2.09(+1)$ & $2.40(+1)$ \\
\hline 7 & 15 & $5.63(+0)$ & $5.47(+0)$ & $5.39(+0)$ & $6.91(+0)$ \\
\hline 7 & 16 & $5.21(+0)$ & $5.78(+0)$ & $5.99(+0)$ & $5.45(+0)$ \\
\hline 8 & 10 & $3.07(+$ & $2.70(+0)$ & 2.22( & $1.96(+0)$ \\
\hline 8 & 11 & 3.6 & $3.41(+0)$ & $2.97(-$ & $2.71(+0)$ \\
\hline 8 & 12 & $5.16(+0)$ & $4.76(+0)$ & $4.16(+0)$ & $3.89(+0)$ \\
\hline 8 & 13 & $7.62(+0)$ & $6.92(+0)$ & $6.35(+0)$ & $6.89(+0)$ \\
\hline 8 & 14 & $3.94(+0)$ & $4.03(+0)$ & $4.54(+0)$ & $5.63(+0)$ \\
\hline 8 & 15 & $1.56(+1)$ & $1.46(+1)$ & $1.31(+1)$ & $1.25(+1)$ \\
\hline 8 & 16 & $8.30(+0)$ & $8.35(+0)$ & $9.10(+0)$ & $1.05(+1)$ \\
\hline 9 & 10 & $3.21(+0)$ & $2.95(+0)$ & $2.37(+0)$ & $1.90(+0)$ \\
\hline 9 & 11 & $4.69(+0)$ & $4.34(+0)$ & $3.38(+0)$ & $2.65(+0)$ \\
\hline 9 & 12 & $5.62(+0)$ & $5.43(+0)$ & $4.73(+0)$ & $4.03(+0)$ \\
\hline 9 & 13 & $1.10(+1)$ & $1.03(+1)$ & $8.63(+0)$ & $7.47(+0)$ \\
\hline 9 & 14 & $7.21(+0)$ & $8.06(+0)$ & $8.83(+0)$ & $9.18(+0)$ \\
\hline 9 & 15 & $1.47(+1)$ & $1.42(+1)$ & $1.32(+1)$ & $1.31(+1)$ \\
\hline 9 & 16 & $2.72(+1)$ & $2.88(+1)$ & $3.36(+1)$ & $4.12(+1)$ \\
\hline
\end{tabular}

Table 4. continued

\begin{tabular}{ccc|cccc}
\hline \multicolumn{2}{l}{ line } & 3.2 & 3.5 & 4.0 & 4.3 \\
\hline 10 & - & 13 & $3.47(+1)$ & $3.34(+1)$ & $3.52(+1)$ & $3.83(+1)$ \\
10 & - & 14 & $2.40(+1)$ & $2.25(+1)$ & $2.11(+1)$ & $1.98(+1)$ \\
10 & - & 15 & $2.40(+1)$ & $2.18(+1)$ & $2.14(+1)$ & $2.25(+1)$ \\
10 & - & 16 & $1.20(+1)$ & $1.09(+1)$ & $9.15(+0)$ & $7.48(+0)$ \\
& & & & & & \\
11 & - & 12 & $2.62(+1)$ & $2.53(+1)$ & $2.73(+1)$ & $3.01(+1)$ \\
11 & - & 14 & $1.84(+1)$ & $1.90(+1)$ & $2.43(+1)$ & $2.94(+1)$ \\
11 & - & 15 & $3.64(+1)$ & $3.26(+1)$ & $2.80(+1)$ & $2.56(+1)$ \\
11 & - & 16 & $2.00(+1)$ & $1.75(+1)$ & $1.45(+1)$ & $1.26(+1)$ \\
& & & & & & \\
12 & - & 15 & $8.92(+2)$ & $9.06(+2)$ & $9.21(+2)$ & $9.15(+2)$ \\
12 & - & 16 & $2.41(+1)$ & $2.24(+1)$ & $1.93(+1)$ & $1.60(+1)$ \\
& & & & & & \\
13 & - & 14 & $1.49(+3)$ & $1.52(+3)$ & $1.54(+3)$ & $1.52(+3)$ \\
13 & - & 16 & $5.62(+1)$ & $5.37(+1)$ & $5.41(+1)$ & $5.46(+1)$ \\
& & & & & & \\
14 & - & 16 & $1.27(+3)$ & $1.29(+3)$ & $1.32(+3)$ & $1.31(+3)$ \\
\hline
\end{tabular}

Table 5. Effective collision strengths for fine-structure transitions in Fe XXVI ions at four temperature points $\log T(\mathrm{~K})=6.0$, $6.5,7.0,7.5$. Line notation corresponds to the level indexes presented in Table 1

\begin{tabular}{rlr|cccc}
\hline \multicolumn{1}{l}{ line } & 6.0 & 6.5 & 7.0 & 7.5 \\
\hline 1 & - & 2 & $1.49(-3)$ & $1.65(-3)$ & $1.74(-3)$ & $1.64(-3)$ \\
1 & - & 3 & $1.14(-3)$ & $1.43(-3)$ & $1.42(-3)$ & $1.18(-3)$ \\
1 & - & 4 & $3.03(-3)$ & $3.67(-3)$ & $3.66(-3)$ & $3.30(-3)$ \\
1 & - & 5 & $3.59(-4)$ & $3.66(-4)$ & $3.52(-4)$ & $3.02(-4)$ \\
1 & - & 6 & $2.79(-4)$ & $2.84(-4)$ & $2.61(-4)$ & $2.11(-4)$ \\
1 & - & 7 & $1.15(-4)$ & $1.17(-4)$ & $8.74(-5)$ & $5.64(-5)$ \\
1 & - & 8 & $6.81(-4)$ & $6.90(-4)$ & $6.74(-4)$ & $5.89(-4)$ \\
1 & - & 9 & $1.64(-4)$ & $1.67(-4)$ & $1.24(-4)$ & $8.03(-5)$ \\
1 & - & 10 & $1.87(-4)$ & $1.61(-4)$ & $1.40(-4)$ & $1.12(-4)$ \\
1 & - & 11 & $1.63(-4)$ & $1.30(-4)$ & $1.05(-4)$ & $7.93(-5)$ \\
1 & - & 12 & $7.15(-5)$ & $5.38(-5)$ & $3.83(-5)$ & $2.54(-5)$ \\
1 & - & 13 & $2.80(-4)$ & $2.66(-4)$ & $2.56(-4)$ & $2.16(-4)$ \\
1 & - & 14 & $2.46(-5)$ & $1.44(-5)$ & $6.55(-6)$ & $2.82(-6)$ \\
1 & - & 15 & $7.82(-5)$ & $6.28(-5)$ & $4.92(-5)$ & $3.46(-5)$ \\
1 & - & 16 & $2.31(-5)$ & $1.39(-5)$ & $6.65(-6)$ & $3.03(-6)$ \\
& & & & & & \\
2 & - & 4 & $2.92(-2)$ & $4.14(-2)$ & $4.02(-2)$ & $2.67(-2)$ \\
2 & - & 5 & $1.36(-2)$ & $1.42(-2)$ & $1.39(-2)$ & $1.19(-2)$ \\
2 & - & 6 & $1.97(-3)$ & $2.19(-3)$ & $1.67(-3)$ & $1.17(-3)$ \\
2 & - & 7 & $4.60(-2)$ & $4.97(-2)$ & $5.66(-2)$ & $5.86(-2)$ \\
2 & - & 8 & $5.49(-3)$ & $5.59(-3)$ & $4.27(-3)$ & $2.84(-3)$ \\
2 & - & 9 & $1.01(-2)$ & $1.00(-2)$ & $7.33(-3)$ & $4.49(-3)$ \\
2 & - & 10 & $3.36(-3)$ & $3.06(-3)$ & $2.77(-3)$ & $2.24(-3)$ \\
2 & - & 11 & $1.19(-3)$ & $8.17(-4)$ & $4.95(-4)$ & $3.03(-4)$ \\
2 & - & 12 & $9.27(-3)$ & $9.27(-3)$ & $1.00(-2)$ & $9.55(-3)$ \\
2 & - & 13 & $2.09(-3)$ & $1.68(-3)$ & $1.18(-3)$ & $7.47(-4)$ \\
2 & - & 14 & $2.58(-3)$ & $2.17(-3)$ & $1.94(-3)$ & $1.73(-3)$ \\
2 & - & 15 & $3.28(-3)$ & $2.65(-3)$ & $1.84(-3)$ & $1.08(-3)$ \\
2 & - & 16 & $1.96(-3)$ & $1.41(-3)$ & $8.65(-4)$ & $4.72(-4)$ \\
\hline
\end{tabular}


Table 5. continued

\begin{tabular}{|c|c|c|c|c|c|}
\hline & line & 6.0 & 6.5 & 7.0 & 7.5 \\
\hline 3 & 5 & $5.20(-3)$ & $5.86(-3)$ & $6.79(-3)$ & $7.43(-3)$ \\
\hline 3 & 6 & $1.26(-2)$ & $1.31(-2)$ & $1.34(-2)$ & $1.19(-2)$ \\
\hline 3 & 7 & $1.13(-2)$ & $1.19(-2)$ & $1.21(-2)$ & $1.12(-2)$ \\
\hline 3 & 8 & $9.34(-3)$ & $1.04(-2)$ & $1.23(-2)$ & $1.37(-2)$ \\
\hline 3 & 9 & $1.63(-2)$ & $1.71(-2)$ & $1.78(-2)$ & $1.66(-2)$ \\
\hline 3 & 10 & $1.85(-3)$ & $1.60(-3)$ & $1.55(-3)$ & $1.48(-3)$ \\
\hline 3 & 11 & $3.16(-3)$ & $2.87(-3)$ & $2.69(-3)$ & $2.26(-3)$ \\
\hline 3 & 12 & $2.68(-3)$ & $2.28(-3)$ & $2.00(-3)$ & $1.62(-3)$ \\
\hline 3 & 13 & $2.85(-3)$ & $2.66(-3)$ & $2.79(-3)$ & $2.77(-3)$ \\
\hline 3 & 14 & $2.02(-3)$ & $1.61(-3)$ & $1.31(-3)$ & $1.02(-3)$ \\
\hline 3 & 15 & $3.70(-3)$ & $3.26(-3)$ & $2.94(-3)$ & $2.43(-3)$ \\
\hline 3 & 16 & $2.46(-3)$ & $2.02(-3)$ & $1.70(-3)$ & $1.33(-3)$ \\
\hline 4 & 5 & $5.85(-3)$ & $5.93(-3)$ & $4.52(-3)$ & $3.04(-3)$ \\
\hline 4 & 6 & $3.91(-3)$ & $4.12(-3)$ & $3.18(-3)$ & $2.35(-3)$ \\
\hline 4 & 7 & $2.25(-2)$ & $2.34(-2)$ & $2.13(-2)$ & $1.79(-2)$ \\
\hline 4 & 8 & $3.29(-2)$ & $3.38(-2)$ & $3.20(-2)$ & $2.68(-2)$ \\
\hline 4 & 9 & $9.40(-2)$ & $1.01(-1)$ & $1.12(-1)$ & $1.13(-1)$ \\
\hline 4 & 10 & $2.28(-3)$ & $1.79(-3)$ & $1.24(-3)$ & $7.80(-4)$ \\
\hline 4 & 11 & $1.78(-3)$ & $1.30(-3)$ & $8.69(-4)$ & $5.72(-4)$ \\
\hline 4 & 12 & $6.12(-3)$ & $5.22(-3)$ & $4.33(-3)$ & $3.28(-3)$ \\
\hline 4 & 13 & $7.95(-3)$ & $7.26(-3)$ & $6.54(-3)$ & $5.21(-3)$ \\
\hline 4 & 14 & $3.66(-3)$ & $2.71(-3)$ & $1.82(-3)$ & $1.18(-3)$ \\
\hline 4 & 15 & $1.95(-2)$ & $1.91(-2)$ & $1.99(-2)$ & $1.85(-2)$ \\
\hline 4 & 16 & $5.60(-3)$ & $4.71(-3)$ & $4.05(-3)$ & $3.44(-3)$ \\
\hline 5 & 8 & $1.30(-1)$ & $1.30(-1)$ & $1.14(-1)$ & $8.91(-2)$ \\
\hline 5 & 9 & $5.34(-2)$ & $5.56(-2)$ & $3.58(-2)$ & $2.01(-2)$ \\
\hline 5 & 10 & $5.93(-2)$ & $5.98(-2)$ & $6.09(-2)$ & $5.25(-2)$ \\
\hline 5 & 11 & $7.38(-3)$ & $6.34(-3)$ & $6.05(-3)$ & $6.11(-3)$ \\
\hline 5 & 12 & $7.53(-2)$ & $8.57(-2)$ & $1.16(-1)$ & $1.31(-1)$ \\
\hline 5 & 13 & $1.21(-2)$ & $1.08(-2)$ & $9.98(-3)$ & $8.63(-3)$ \\
\hline 5 & 14 & $8.09(-2)$ & $8.51(-2)$ & $9.54(-2)$ & $9.08(-2)$ \\
\hline 5 & 15 & $1.82(-2)$ & $1.52(-2)$ & $1.21(-2)$ & $9.06(-3)$ \\
\hline 5 & 16 & $2.23(-2)$ & $1.71(-2)$ & $1.24(-2)$ & $8.78(-3)$ \\
\hline 6 & 7 & $9.08(-2)$ & $9.26(-2)$ & $7.76(-2)$ & $5.84(-2)$ \\
\hline 6 & 9 & $1.39(-1)$ & $1.40(-1)$ & $1.17(-1)$ & $8.81(-2)$ \\
\hline 6 & 10 & $1.47(-2)$ & $1.65(-2)$ & $2.29(-2)$ & $2.75(-2)$ \\
\hline 6 & 11 & $5.83(-2)$ & $5.90(-2)$ & $6.07(-2)$ & $5.28(-2)$ \\
\hline 6 & 12 & $2.86(-2)$ & $2.98(-2)$ & $3.35(-2)$ & $3.22(-2)$ \\
\hline 6 & 13 & $2.42(-2)$ & $2.81(-2)$ & $4.11(-2)$ & $5.05(-2)$ \\
\hline 6 & 14 & $3.13(-2)$ & $2.94(-2)$ & $2.77(-2)$ & $2.28(-2)$ \\
\hline 6 & 15 & $4.26(-2)$ & $4.45(-2)$ & $5.00(-2)$ & $4.80(-2)$ \\
\hline 6 & 16 & $4.18(-2)$ & $3.90(-2)$ & $3.68(-2)$ & $3.04(-2)$ \\
\hline
\end{tabular}

Table 5. continued

\begin{tabular}{|c|c|c|c|c|c|c|}
\hline & lin & & 6.0 & 6.5 & 7.0 & 7.5 \\
\hline 7 & - & 9 & $2.06(-1)$ & $2.15(-1)$ & $1.35(-1)$ & $7.06(-2)$ \\
\hline 7 & - & 10 & $1.29(-2)$ & $1.06(-2)$ & $8.70(-3)$ & $7.34(-3)$ \\
\hline 7 & - & 11 & $8.42(-3)$ & $6.26(-3)$ & $4.17(-3)$ & $2.80(-3)$ \\
\hline 7 & - & 12 & $1.27(-1)$ & $1.23(-1)$ & $1.20(-1)$ & $1.01(-1)$ \\
\hline 7 & - & 13 & $1.75(-2)$ & $1.27(-2)$ & $7.87(-3)$ & $4.86(-3)$ \\
\hline 7 & - & 14 & $5.02(-1)$ & $5.44(-1)$ & $6.55(-1)$ & $6.76(-1)$ \\
\hline 7 & - & 15 & $3.43(-2)$ & $2.46(-2)$ & $1.52(-2)$ & $8.94(-3)$ \\
\hline 7 & - & 16 & $5.64(-2)$ & $4.05(-2)$ & $2.46(-2)$ & $1.39(-2)$ \\
\hline 8 & - & 10 & $1.23(-2)$ & $1.12(-2)$ & $1.06(-2)$ & $9.42(-3)$ \\
\hline 8 & - & 11 & $1.16(-2)$ & $1.05(-2)$ & $1.17(-2)$ & $1.28(-2)$ \\
\hline 8 & - & 12 & $3.85(-2)$ & $3.72(-2)$ & $3.98(-2)$ & $3.92(-2)$ \\
\hline 8 & - & 13 & $1.28(-1)$ & $1.29(-1)$ & $1.32(-1)$ & $1.15(-1)$ \\
\hline 8 & - & 14 & $5.30(-2)$ & $4.73(-2)$ & $4.41(-2)$ & $3.79(-2)$ \\
\hline 8 & - & 15 & $1.58(-1)$ & $1.76(-1)$ & $2.29(-1)$ & $2.55(-1)$ \\
\hline 8 & - & 16 & $1.59(-1)$ & $1.61(-1)$ & $1.76(-1)$ & $1.65(-1)$ \\
\hline 9 & - & 10 & $1.13(-2)$ & $8.11(-3)$ & $4.90(-3)$ & $2.82(-3)$ \\
\hline 9 & - & 11 & $1.20(-2)$ & $9.12(-3)$ & $6.21(-3)$ & $4.22(-3)$ \\
\hline 9 & - & 12 & $3.39(-2)$ & $2.43(-2)$ & $1.51(-2)$ & $9.01(-3)$ \\
\hline 9 & - & 13 & $3.18(-2)$ & $2.50(-2)$ & $1.92(-2)$ & $1.51(-2)$ \\
\hline 9 & - & 14 & $9.75(-2)$ & $8.27(-2)$ & $7.35(-2)$ & $6.35(-2)$ \\
\hline 9 & - & 15 & $2.11(-1)$ & $2.03(-1)$ & $1.91(-1)$ & $1.58(-1)$ \\
\hline 9 & - & 16 & $7.63(-1)$ & $8.25(-1)$ & $9.96(-1)$ & $9.84(-1)$ \\
\hline 10 & - & 13 & $4.17(-1)$ & $3.87(-1)$ & $3.56(-1)$ & $2.83(-1)$ \\
\hline 10 & - & 14 & $2.99(-1)$ & $2.64(-1)$ & $2.29(-1)$ & $1.78(-1)$ \\
\hline 10 & - & 15 & $1.01(-1)$ & $8.11(-2)$ & $6.60(-2)$ & $5.22(-2)$ \\
\hline 10 & - & 16 & $9.93(-2)$ & $6.36(-2)$ & $3.49(-2)$ & $1.98(-2)$ \\
\hline 11 & - & 12 & $3.36(-1)$ & $3.13(-1)$ & $2.87(-1)$ & $2.27(-1)$ \\
\hline 11 & - & 14 & $8.65(-2)$ & $6.23(-2)$ & $4.14(-2)$ & $2.84(-2)$ \\
\hline 11 & - & 15 & $5.06(-1)$ & $4.72(-1)$ & $4.33(-1)$ & $3.43(-1)$ \\
\hline 11 & - & 16 & $1.17(-1)$ & $8.91(-2)$ & $5.86(-2)$ & $3.92(-2)$ \\
\hline 12 & - & 15 & $3.42(-1)$ & $2.66(-1)$ & $2.00(-1)$ & $1.44(-1)$ \\
\hline 12 & - & 16 & $2.56(-1)$ & $1.55(-1)$ & $7.75(-2)$ & $4.00(-2)$ \\
\hline 13 & - & 14 & $2.30(-1)$ & $1.68(-1)$ & $1.14(-1)$ & $7.71(-2)$ \\
\hline 13 & - & 16 & $6.04(-1)$ & $5.17(-1)$ & $4.26(-1)$ & $3.20(-1)$ \\
\hline 14 & - & 16 & $7.37(-1)$ & $4.55(-1)$ & $2.33(-1)$ & $1.19(-1)$ \\
\hline
\end{tabular}

